\title{
Monocytic cell junction proteins serve important roles in atherosclerosis via the endoglin pathway
}

\author{
LINA CHEN, ZHONGLIANG CHEN, MENGHUA GE, OUSHAN TANG, \\ YINHONG CHENG, HAOLIANG ZHOU, YU SHEN and FENGMING QIN
}

\author{
Department of Cardiology, Shaoxing Second Hospital, Shaoxing, Zhejiang 312000, P.R. China
}

Received August 19, 2016; Accepted June 15, 2017

DOI: $10.3892 / \mathrm{mmr} .2017 .7444$

\begin{abstract}
The formation of atherosclerosis is recognized to be caused by multiple factors including pathogenesis in monocytes during inflammation. The current study provided evidence that monocytic junctions were significantly altered in patients with atherosclerosis, which suggested an association between cell junctions and atherosclerosis. Claudin-1, occludin-1 and ZO-1 were significantly enhanced in atherosclerosis, indicating that the tight junction pathway was activated during the pathogenesis of atherosclerosis. In addition, the gene expression of 5 connexin members involved in the gap junction pathway were quantified, indicating that connexin 43 and 46 were significantly up-regulated in atherosclerosis. Furthermore, inflammatory factors including endoglin and SMAD were observed, suggesting that immune regulative factors were down-regulated in this pathway. Silicon-based analysis additionally identified that connexins and tight junctions were altered in association with monocytic inflammation regulations, endoglin pathway. The results imply that reduced expression of the immune regulation pathway in monocytes is correlated with the generation of gap junctions and tight junctions which serve important roles in atherosclerosis.
\end{abstract}

\section{Introduction}

Atherosclerosis is currently recognized as the outcome of the formation of multiple atheromatous plaques in the arteries, which has been characterized as arterial chronic degeneration and inflammation (1). Recruitment of leukocytes from the blood to the arterial wall is an early and critical step in the inflammatory cascade $(1,2)$. Collagen and proteoglycans then accumulate, which results in arterial hardening and thickening, leading to a loss of flexibility of the arteries (3). Once the

Correspondence to: Dr Lina Chen, Department of Cardiology, Shaoxing Second Hospital, 141 Yanan Road, Shaoxing, Zhejiang 312000, P.R. China

E-mail: linachen130@163.com

Key words: atherosclerosis, endoglin pathway, monocytic cell junction proteins disease is established, it may manifest with angina pectoris, myocardial infarction and other potentially fatal diseases, thus has a high mortality rate (3-5). The process and cause of arterial thickening is complex, including the involvement of inflammatory cells (epithelial cells, monocytes, macrophages, smooth muscle and platelets), molecular factors (lipoprotein, growth hormones, cholesterol, fat or collagen) and cytokines (interleukins, tumor necrosis factors and chemokines) $(3,6)$.

Gap junctions act a bridge for communication between two adjacent cells via connexins, arranged in a hexamer membrane structure (1,7-9). The exchange of information and energy material are between adjacent cells is mediated through gap junction intercellular space connection communication (gap junction intercellular communication) (7). These communication channels allow for the transport of ions, small molecular metabolites and secondary signaling molecules, allowing material exchange between cells and electrical coupling $(1,8)$.

Studies have demonstrated that the expression of the junction proteins, including tight junction proteins and gap junction proteins is closely associated with atherosclerosis. Tight junction protein (TJP) is an adhesive structure between the cells $(10,11)$. Cell adhesion is involved in maintaining stability of organizational homeostasis, and is a factor for cellular movement, regulation of permeability, cell differentiation and cell proliferation $(2,12)$. Tight junctions is composed of a group of proteins including the claudin family, junction adhesion molecules (JAMs) and the closed small ring protein $\mathrm{ZO}$ family $(4,12,13)$; previous studies have demonstrated that these proteins do not take part in gap junction formation, however facilitate the release of small functional molecules such as adenosine triphosphate (ATP) $(1,3,8)$. Connexin 43 (Cx43) is predominantly expressed in myocardial tissue, macrophages, connective tissue cells, endothelial cells and smooth muscle cells $(14,15)$. Cx43 forms a half channel and then is transported to the plasma membrane by the Golgi apparatus. Previous studies have demonstrated that is $\mathrm{Cx} 43$ is essential for the differentiation and development of the heart; abnormal expression of Cx43 can lead to a variety of cardiovascular diseases including congenital malformation of the heart $(6,8,9,14,15)$.

It has been reported that the upregulation of $\mathrm{Cx} 43$ expression enhanced monocyte-endothelial adhesion and vice versa when downregulated. This mechanism was associated with Cx43-induced vascular cell adhesion molecules and intercellular cell adhesion molecules suggesting that local regulation 
Table I. Primers designed using Primer 6.0 software and synthesized from Generay Biotech.

\begin{tabular}{|c|c|c|}
\hline Gene name & Primer sequence ( $5^{\prime}$ to $\left.3^{\prime}\right)$ & Amplicon size/NCBI.NM \\
\hline H-GAPDH-157 bp-F & GAGTCCACTGGCGTCTTCAC & $157 \mathrm{bp}$ \\
\hline H-GAPDH-157 bp-R & TGCTGATGATCTTGAGGCTGTT & NM_001256799.2 \\
\hline $\mathrm{H}-\mathrm{CX} 37-\mathrm{F}$ & AGTTCCTCTTCGTCAGCACAC & $239 \mathrm{bp}$ \\
\hline $\mathrm{H}-\mathrm{CX} 37-\mathrm{R}$ & GAGCACACTGGCGACATAGG & NM_002060.2 \\
\hline $\mathrm{H}-\mathrm{CX} 40-\mathrm{F}$ & TGGAAGAAGATCAGACAGCGATT & $218 \mathrm{bp}$ \\
\hline $\mathrm{H}-\mathrm{CX} 40-\mathrm{R}$ & CCTCGTACTTGCTCGGTGAC & NM_005266.6 \\
\hline $\mathrm{H}-\mathrm{CX} 43-\mathrm{F}$ & CTGGTGGTGTCCTTGGTGTC & $182 \mathrm{bp}$ \\
\hline $\mathrm{H}-\mathrm{CX} 43-\mathrm{R}$ & GGTGAGGAGCAGCCATTGAA & NM_000165.4 \\
\hline $\mathrm{H}-\mathrm{CX} 45-\mathrm{F}$ & ACCGAACTGTCCAATGCTAAGA & $142 \mathrm{bp}$ \\
\hline $\mathrm{H}-\mathrm{CX} 45-\mathrm{R}$ & AGCGTTCCTGAGCCATCCT & NM_001080383.1 \\
\hline $\mathrm{H}-\mathrm{CX} 46-\mathrm{F}$ & TGTTCATCTTCCGCATCTTGGT & $119 \mathrm{bp}$ \\
\hline $\mathrm{H}-\mathrm{CX} 46-\mathrm{R}$ & CCTGTCGTAGCAGACGTTCTC & NM_021954.3 \\
\hline H-Claudin-1-F & AGGTCTTGCCGCCTTGGTA & $173 \mathrm{bp}$ \\
\hline H-Claudin-1-R & GACAGGAACAGGAGAGCAGTG & NM_001307.5 \\
\hline H-Endoglin-F & CGACGCCAACCACAACAT & $156 \mathrm{bp}$ \\
\hline H-Endoglin-R & ACGAAGGATGCCACAATGC & NM_000118.3 \\
\hline H-Occludin-1-F & TCGCTGCCAATGCTCATCTG & 206 bp \\
\hline H-Occludin-1-R & GCCTCCAAGGAAGAGACTGAAG & NM_005985.3 \\
\hline H-SMAD-F & CATGCCACTCAACGCCACTT & 295 bp \\
\hline H-SMAD-R & AACCGCCTGAACATCTCCTCT & NM_005900.2 \\
\hline $\mathrm{H}-\mathrm{ZO}-1-\mathrm{F}$ & GCGGATGGTGCTACAAGTGAT & $138 \mathrm{bp}$ \\
\hline $\mathrm{H}-\mathrm{ZO}-1-\mathrm{R}$ & GCCTTCTGTGTCTGTGTCTTCA & NM_001301025.1 \\
\hline
\end{tabular}

GAPDH, glyceraldehyde 3-phosphate dehydrogenase; bp, base pairs; F, forward; R, reverse; SMAD, mothers against decapentaplegic homolog; ZO-1, tight junction protein 1 .

of endothelial $\mathrm{Cx} 43$ expression within the vasculature regulates monocyte endothelial adhesion and the development of atherosclerosis (16).

A previous study indicated that circulating monocytes expressing low levels of $\mathrm{Cx} 37$ protect against atherosclerosis by regulating monocyte adhesion, however the expression of other connexins remains low (17). However upon stimulation with cytokines including tumor necrosis factor (TNF) $\alpha$ and interferon $\gamma$, human blood monocytes were demonstrated to express high levels of Cx43 (18).

A previous study focused on the association between tight junction and atherosclerosis progression, observing that blocking myosin light chain kinase by inhibitor ML7 improved vascular endothelial dysfunction and atherosclerosis (19). This mechanism is explained by the regulation of TJP1 (ZO-1) and occludins in the atherosclerosis model, which implies overexpression of tight junctions may serve important roles in the pathogenesis.

It is currently recognized that monocytes originate from progenitors in the bone marrow and they reach the circulation as two major subgroups, the classical CD14(high)CD16 monocytes and the CD14(low)CD16 ${ }^{+}$monocytes (20).

Endoglin [CD105, type III transforming growth factor (TGF)- $\beta$ receptor] and mothers against decapentaplegic homolog (SMAD) pathways were identified to be involved in the negative regulation pathway of inflammation resulting in
Table II. Quantitative polymerase chain reaction components $(20 \mu \mathrm{l})$.

\begin{tabular}{lc}
\hline PCR components & Volume, $\mu 1$ \\
\hline RNase-free $\mathrm{H}_{2} \mathrm{O}$ & 7.2 \\
2X Realtime PCR master mix & 10 \\
Forward primer, $10 \mu \mathrm{M}$ & 0.4 \\
Reverse primer, $10 \mu \mathrm{M}$ & 0.4 \\
cDNA template & 2 \\
\hline
\end{tabular}

the release of TGF- $\beta$, interleukin 4 and other immune suppressive proteins (21). In addition, the role of endoglin in the monocyte circulation system, including cell-mediated vascular repair, has been well-studied (22). Therefore, it is critical to understand the immunological homeostasis of monocytes and possible correlation between cellular junctions and the endoglin/SMAD pathway during atherosclerosis.

CD14 marker-positive isolation is an optimal way to study monocytic gap junctions and tight junctions from patients and normal controls. In the present study, the focus was predominantly on the expression level of key gap junction proteins (Cx37, Cx40, Cx43, Cx45 and Cx46), TJPs (claudin-1, occludin-1 and ZO-1), and the inflammation regulating genes, 

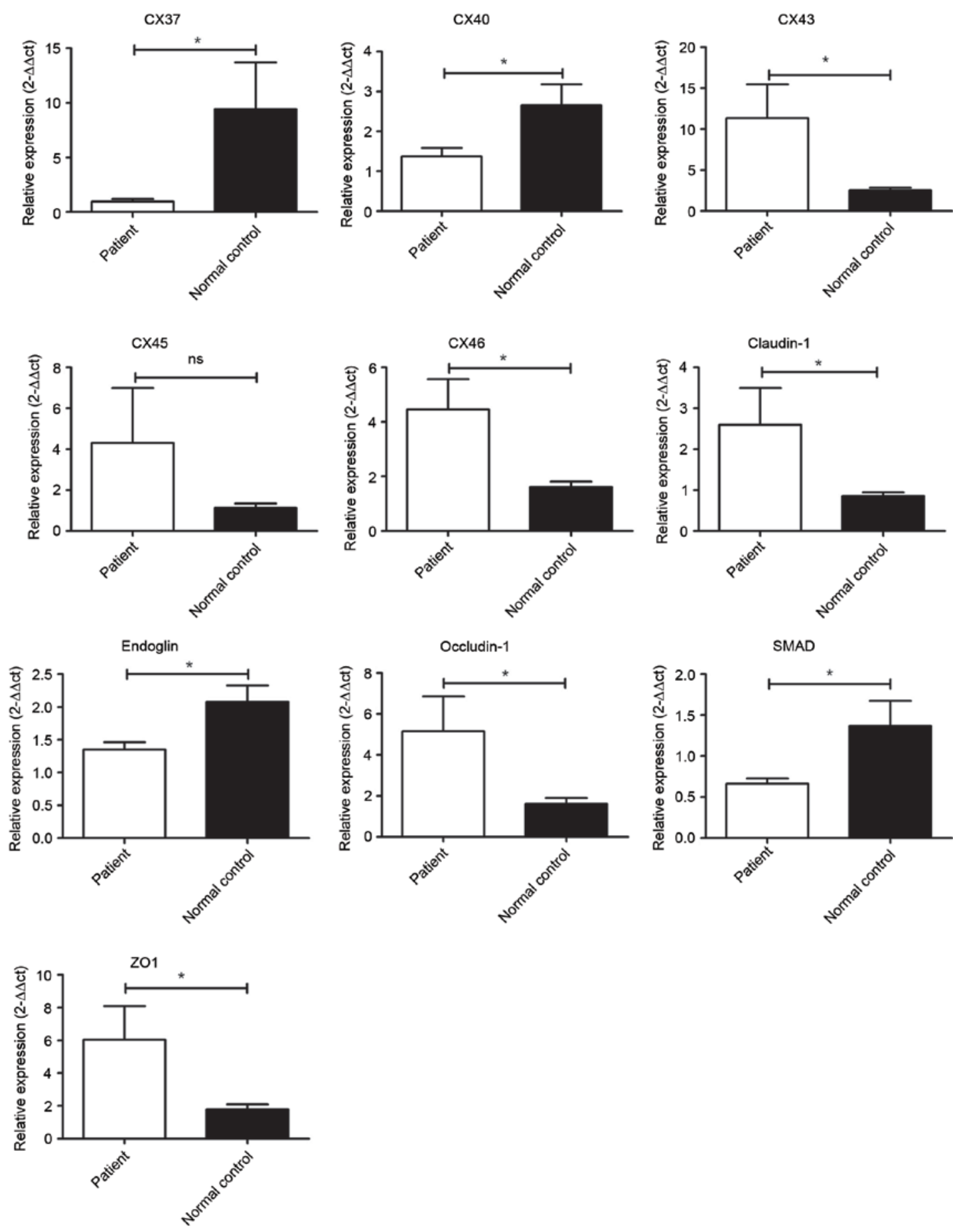

Figure 1. Transcriptional analysis of genes promoting atherosclerosis. Cx43, Cx45, Cx46, claudin-1, occludin-1 and ZO-1 were significantly elevated in patients . However, Cx37, Cx40, endoglin and SMAD1 were downregulated in patients. "P<0.05, patient compared with normal control. ZO-1, tight junction protein 1; SMAD1, mothers against decapentaplegic homolog 1.

endoglin and SMAD1, in atherosclerotic patients and normal controls. Further combination analysis of these genes with public datasets were performed to draw a more comprehensive correlation between atherosclerosis and junction proteins, and how they act on immune regulatory pathways.

\section{Materials and methods}

Patients and samples. The subjects were divided into two groups with the results of the coronary angiogram. A total of
25 outpatients with indications of stent implantation and 25 normal controls that were under routine physical examination were admitted into Shaoxing Second Hospital (Shaoxing, China). The protocols were reviewed and approved by the Animal Ethical and Welfare Committee of Shaoxing Second Hospital. Informed consent was obtained from each patient. CD14+ fluorescent antibody at $5 \mu \mathrm{l} /$ million cells (cat. no. 367115; BioLegend, San Diego, CA, USA) and flow cytometry (BD Biosciences, Franklin Lakes, NJ, USA) were applied on isolated monocytes from peripheral blood mononuclear cells. 


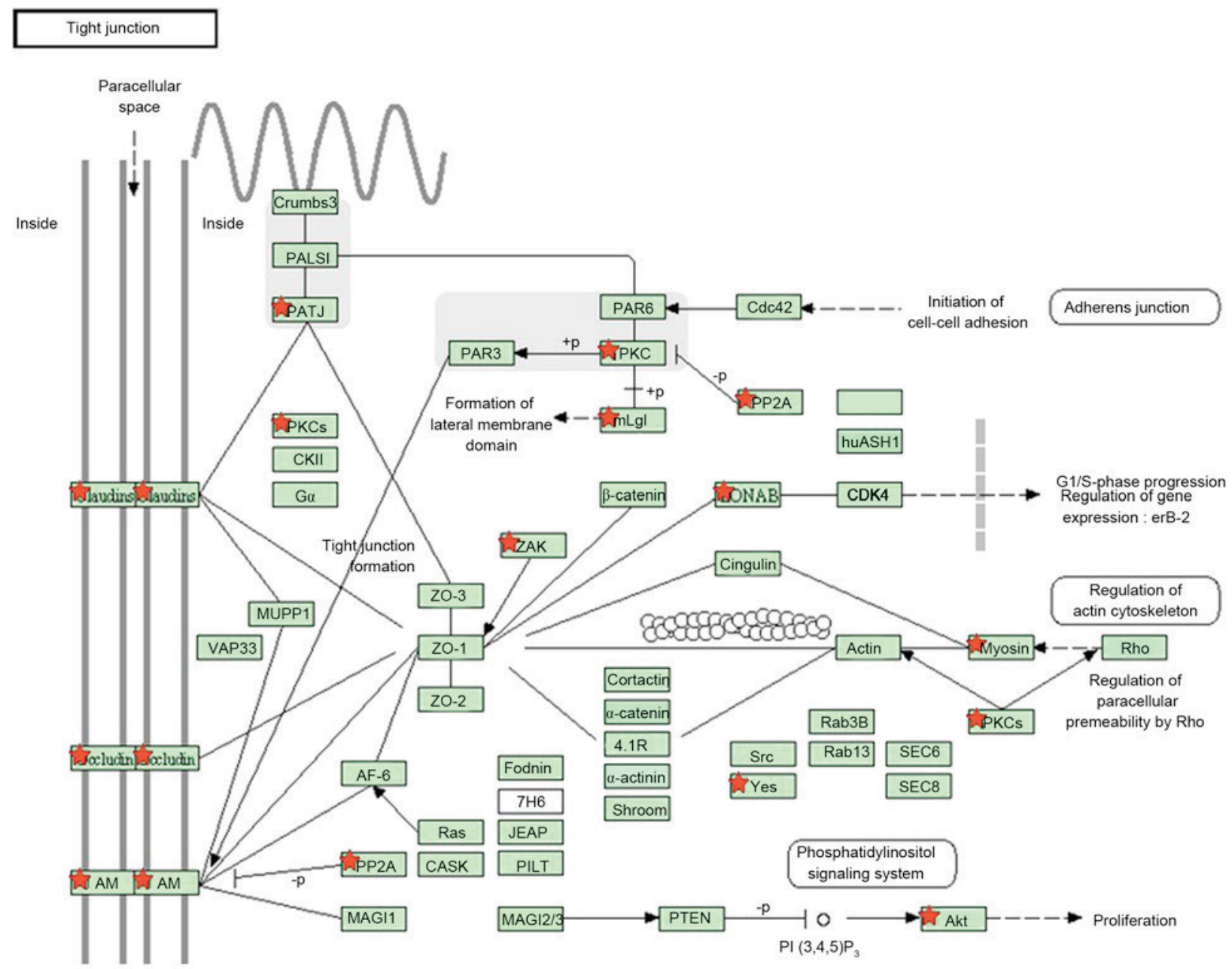

Figure 2. Tight junction pathway of patients with CHD. Genes marked with red stars indicate those exhibiting differential expression.

Reverse transcription-quantitative polymerase chain reaction $(R T-q P C R)$. Total RNA from the monocytes of patients was extracted using TRIzol (cat. no. 15596018, Thermo Fisher Scientific, Inc., Waltham, MA, USA) reagent. The quantity and quality of RNA were confirmed using a NanoDrop 1000 thermocycler (NanoDrop; Thermo Fisher Scientific, Inc.) and 1.0\% agarose (Hushi, Shanghai, China; cat. no. CAS:9002-18-0) electrophoresis. The primers were designed using Primer 6.0 software (Premier Biosoft International, Palo Alto, CA, USA) and were synthesized by Shanghai Generay Biotech Co., Ltd. (Shanghai, China) (Table I).

For gene specific quantitation, reverse transcription was performed based on the specification of the ReverTra Ace qPCR RT kit (Toyobo Co., Ltd., Osaka, Japan) to synthesize the first strand of cDNA and RT-qPCR were conducted on FTC-3000 (Funglyn Biotech, Inc., Richmond Hill, ON, Canada) with SYBR Green Fast qPCR kit (Kapa Biosystems, Inc., Wilmington, MA, USA) (Table II). Thermal cycling parameters were set as follows: $95^{\circ} \mathrm{C}$ for $3 \mathrm{~min}$ (enzyme activation), the next stage was repeated 40 times; $95^{\circ} \mathrm{C}$ for $5 \mathrm{sec}$ (denaturation), and $60^{\circ} \mathrm{C}$ for $30 \mathrm{sec}$ (annealing/extension/data acquisition). Data were analyzed by the $2^{-\Delta \Delta \mathrm{Cq}}$ algorithm (23).

Combined analysis of microarray data from coronary heart disease (CHD) study. The microarray dataset GSE71226 was downloaded from NCBI Gene Expression Omnibus (https://www.ncbi.nlm.nih.gov/geo/). This dataset included the microarray gene expression profiling of peripheral blood of three Chinese patients with CHD and three Chinese healthy controls. The raw data were normalized with $\mathrm{R}$ project limma packages in Bioconductor (https://www.bioconductor.org/) with default settings. Fold change of gene expression and corresponding t-test $\mathrm{P}$-values were calculated between patients with CHD and healthy individuals. Differentially expressed genes (DEGs) were defined as genes that met the criteria of fold change value $>1.5$ and t-test $\mathrm{P}<0.05$. DEGs were used with the Kyoto Encyclopedia of Genes and Genomes (KEGG) pathway database (http://www.genome.jp/kegg/) to determine the biological function of these DEGs. Enriched pathways were determined by those yielding a significant value from Fisher's exact test $(\mathrm{P}<0.05)$.

Statistical analysis. Student's t-test was used to filter the DEGs between control groups and patient groups (Fold change value $>1.5$ and t-test $\mathrm{P}<0.05$ ), and a Fisher's exact test was performed to determine the enriched pathways. Statistical analyses were performed using GraphPad Prism 5 software (GraphPad Software, Inc., La Jolla, CA, USA) and SPSS version 17.0 statistical software program/package (SPSS, Inc., Chicago, IL, USA). 


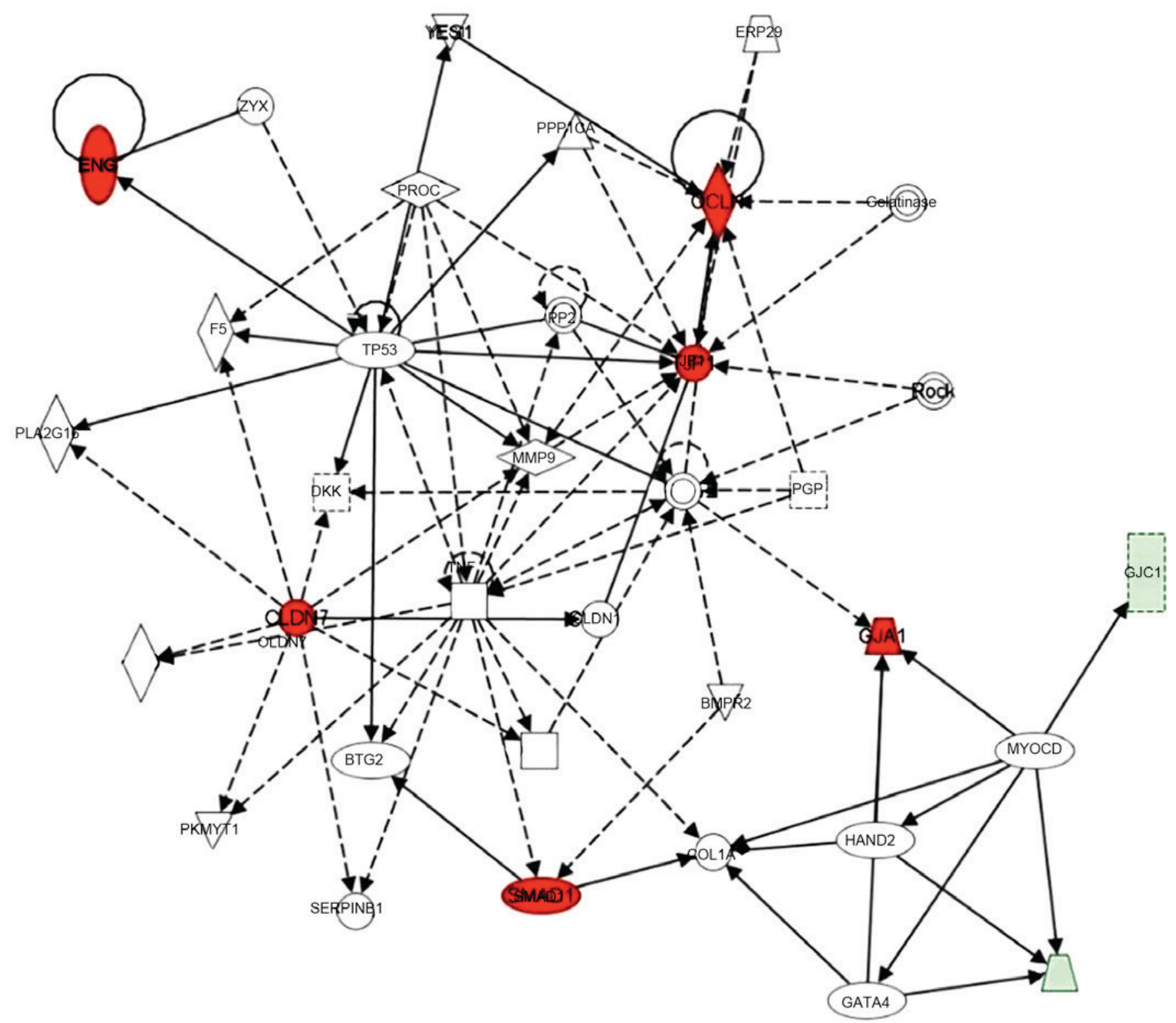

Figure 3. Ingenuity Pathway Analysis gene-gene interaction network analysis, where red circles indicate upregulated genes and green represented genes that were downregulated in patients with CHD.

\section{Results}

Gene expression quantification of cell junction genes in CHD patients. To further clarify the important role of the cell junction in the pathogenesis of atherosclerosis, eight genes that were involved in the gap junction and tight junction pathways in CHD patients were investigated by RT-qPCR. As presented in Fig. 1, Cx43 and Cx46, the major components of the gap junctions localized on the cell surface, were significantly increased $(\mathrm{P}<0.05)$ in patients with $\mathrm{CHD}$. In addition, $\mathrm{Cx} 45$ was marginally enhanced in patients with CHD ( $\mathrm{P}>0.05)$. However, the levels of $\mathrm{Cx} 37$ and $\mathrm{Cx} 40$ were significantly downregulated in patients with CHD compared with normal controls $(\mathrm{P}<0.05)$. In terms of TJPs, claudin-1, occludin-1 and ZO-1 were all significantly increased in in patients with $\mathrm{CHD}$ $(\mathrm{P}<0.05)$.

In addition, expression of endoglin and SMAD1 were significantly reduced in patients with CHD.
Microarray data analysis of CHD. To obtain genes associated with CHD, DEGs were identified by calculating the gene expression fold change and t-test $\mathrm{P}$-value from peripheral blood monocytic cells between patients and healthy individuals. In total, 1,774 genes were identified as DEGs. All DEGs identified were used with the KEGG database for pathway enrichment analysis. The tight junction pathway was significantly enriched and 18 DEGs were involved in this pathway, which includes the claudin-1 and occludin- 1 genes. The results indicated that there was an alteration of the tight junction pathway in patients with CHD (Fig. 2).

A gene interaction network was constructed base on these significantly regulated genes using Ingenuity Pathway Analysis (IPA) tools. Claudin-7, occludin-1, ZO-1 and SMAD1 were identified as hub genes in this network. TNF was also involved in this network, and the myocardin gene connected three connexin family members ( $\mathrm{Cx} 43$ as a core gene, and $\mathrm{Cx} 33$ and $\mathrm{Cx} 45$; in red circles). In addition, chemokine 
(C-X3-C motif) ligand 1, matrix metalloproteinase 9, tumor protein 53 and several other genes were also involved in this network (Fig. 3).

\section{Discussion}

The present study provided evidence that the key factor of cell junctions were significantly altered in patients with CHD with atherosclerosis, which indicated an association between cell junctions and atherosclerosis. The claudin-1, occludin-1 and ZO-1 were significantly enhanced in atherosclerosis, indicating that tight junction pathway was activated during the pathogenesis of atherosclerosis. In addition, the gene expression of 5 connexin members involved in the gap junction pathway were quantified, Cx43 and Cx46 were significantly upregulated in atherosclerosis. Combined in silico lab analysis also agreed with the evidence that connexins and tight junctions were altered in association with monocytic inflammation regulations. Further inflammatory factors including endoglin and SMAD were evaluated to be downregulated in the pathway. The presence of different cell junction contacts is an essential characteristic of different cell systems, with a high degree of cell-to-cell integration and communication (24,25). It can be hypothesized that the disturbances in functioning of gap junctions may be the cause of the disintegration of the cellular network in atherosclerotic disease.

The importance of gap junction-mediated intercellular communication in the development of the pathogenesis of atherosclerosis has been discussed previously $(15,26)$. A previous study indicated that high levels of $\mathrm{Cx} 43$ expression were associated with reduced contractile ability and an increase in the synthetic phenotype in smooth muscle cells (27). It was previously reported that the upregulation of $\mathrm{Cx} 43$ enhanced monocyte-endothelial adhesion and therefore the suggested mechanism was that $\mathrm{Cx} 43$ induced an increase in monocyte adherance onto vascular endothelial cells during the development of atherosclerosis (16). The results of the current study from both the wet lab and dry lab supported this hypothesis, that Cx43 was upregulated in atherosclerotic patients. In addition, Cx45 and Cx46 were identified to present with the same pattern as $\mathrm{Cx} 43$, exhibiting synergistic function or association. Cx37 and $\mathrm{Cx} 40$ were additionally observed to exhibit reduced expression in monocytes in the current study, resulting in altered ATP release during the monocyte-endothelial adhesion bioprocess.

Intercellular tight junctions serve important roles in the stability of endothelial barriers as they regulate paracellular permeability. They are composed of families including claudins, occludins and JAMs $(2,12)$. ZO-1, as a junctional adaptor protein, in addition to the transmembrane proteins of the claudin and JAM families, interacts with multiple junction components and transduces molecules in the signaling pathways $(24,25)$.

As reported by Collins et al (28), increased levels of occludin and ZO-1 and increased localization to cell-cell junctions resulted in a parallel reduction in transendothelial protein permeability. The changes of TJP expression were also accompanied by alterations in their phosphorylation state. Cheng et al (19) reported that the myosin light chain kinase (MLCK) inhibitor ML7 was used to improve atherosclerosis by regulating the expression of $\mathrm{ZO}-1$ and occludin through
MLCK and MLC phosphorylation in a high-fat diet fed rabbit model (29).

In the current study, a higher expression level of TJPs was observed, which is in agreement with the previous findings in animal model studies $(19,28,29)$. Increased TJP expression may contribute to more stable contact after monocytes adhere to endothelia during the initial steps of atherosclerotic pathogenesis.

In the investigation of the mechanisms involved, it was identified that the TGF- $\beta$ /endoglin and SMAD pathway were suppressed in patients with atherosclerosis. The pathway was reported to have a negative correlation between atherosclerosis and its activation. Therefore it was hypothesized that reduced expression of this immune regulatory pathway is correlated with the generation of gap junctions and tight junctions. However, further validation of a causal association between the endoglin pathway and TJPs or gap junction proteins is required.

In conclusion, the present study demonstrated that cell junction pathways including tight junctions and gap junctions are actively involved in the progression of atherosclerosis.

Increased levels of TJPs, claudin-1, occludin-1 and ZO-1, and gap junction proteins $\mathrm{Cx} 43$ and $\mathrm{Cx} 46$ were detected with significance in atherosclerosis. The endoglin and SMAD inflammatory regulatory pathways were suppressed in patients with atherosclerosis, and silicon-based data analysis additionally confirmed core roles of gap junctions and tight junctions in monocytic inflammatory regulation. Accordingly, the genes identified in the current study may represent a possible target for prevention of atherosclerosis by blocking monocytic cell junctions.

\section{References}

1. Scheckenbach KE, Crespin S, Kwak BR and Chanson M: Connexin channel-dependent signaling pathways in inflammation. J Vascular Res 48: 91-103, 2011.

2. Berardi DE and Tarbell JM: Stretch and shear interactions affect intercellular junction protein expression and turnover in endothelial cells. Cell Mol Bioeng 2: 320-331, 2009.

3. Morel S, Burnier L and Kwak BR: Connexins participate in the initiation and progression of atherosclerosis. Semin Immunopathol 31: 49-61, 2009.

4. Zhou T, He Q, Tong Y, Zhan R, Xu F, Fan D, Guo X, Han H, Qin S and Chui D: Phospholipid transfer protein (PLTP) deficiency impaired blood-brain barrier integrity by increasing cerebrovascular oxidative stress. Biochem Biophys Res Commun 445: 352-356, 2014

5. Wong CW, Burger F, Pelli G, Mach F and Kwak BR: Dual benefit of reduced $\mathrm{Cx} 43$ on atherosclerosis in LDL receptor-deficient mice. Cell Commun Adhes 10: 395-400, 2003.

6. Wei JM, Wang X, Gong H, Shi YJ and Zou Y: Ginkgo suppresses atherosclerosis through downregulating the expression of connexin 43 in rabbits. Arch Med Sci 9: 340-346, 2013.

7. Ren Q, Riquelme MA, Xu J, Yan X, Nicholson BJ, Gu S and Jiang JX: Cataract-causing mutation of human connexin 46 impairs gap junction, but increases hemichannel function and cell death. PLoS One 8: e74732, 2013.

8. Retamal MA: Connexin and Pannexin hemichannels are regulated by redox potential. Front Physiol 5: 80, 2014.

9. Yuan D, Wang Q, Wu D, Yu M, Zhang S, Li L, Tao L and Harris AL: Monocyte-endothelial adhesion is modulated by Cx43-stimulated ATP release from monocytes. Biochem Biophys Res Commun 420: 536-541, 2012.

10. Ba J, Peng H, Chen Y and Gao Y: Effects and mechanism analysis of vascular endothelial growth factor and salvianolic acid B on 125I-low density lipoprotein permeability of the rabbit aortary endothelial cells. Cell Biochem Biophys 70: 1533-1538, 2014.

11. Telo P, Lostaglio $S$ and Dejana E: Structure of intercellular junctions in the endothelium. Therapie 52: 395-398, 1997. 
12. Lehman DM, Leach RJ, Johnson-Pais T, Hamlington J, Fowler S, Almasy L, Duggirala R, Stern MP and Abboud HE: Evaluation of tight junction protein 1 encoding zona occludens 1 as a candidate gene for albuminuria in a Mexican American population. Exp Clin Endocrinol Diabetes 114: 432-437, 2006.

13. Gan H, Wang G, Hao Q, Wang QJ and Tang H: Protein kinase D promotes airway epithelial barrier dysfunction and permeability through down-regulation of claudin-1. J Biol Chem 289: 20489, 2014.

14. Polacek D, Bech F, McKinsey JF and Davies PF: Connexin43 gene expression in the rabbit arterial wall: Effects of hypercholesterolemia, balloon injury and their combination. J Vasc Res 34: 19-30, 1997.

15. Chadjichristos CE, Matter CM, Roth I, Sutter E, Pelli G, Lüscher TF, Chanson M and Kwak BR: Reduced connexin43 expression limits neointima formation after balloon distension injury in hypercholesterolemic mice. Circulation 113: 2835-2843, 2006.

16. Yuan D, Sun G, Zhang R, Luo C, Ge M, Luo G and Hei Z: Connexin 43 expressed in endothelial cells modulates monocyteendothelial adhesion by regulating cell adhesion proteins. Mol Med Rep 12: 7146-7152, 2015.

17. Kameritsch P, Pogoda K and Pohl U: Channel-independent influence of connexin 43 on cell migration. Biochim Biophys Acta 1818: 1993-2001, 2012.

18. Morel S and Kwak BR: Roles of connexins in atherosclerosis and ischemia-reperfusion injury. Curr Pharm Biotechnol 13: 17-26, 2012.

19. Cheng X, Wang X, Wan Y, Zhou Q, Zhu H and Wang Y: Myosin light chain kinase inhibitor ML7 improves vascular endothelial dysfunction via tight junction regulation in a rabbit model of atherosclerosis. Mol Med Rep 12: 4109-4116, 2015.

20. Decano JL, Mattson PC and Aikawa M: Macrophages in Vascular Inflammation: Origins and Functions. Curr Atheroscler Rep 18: 34, 2016.
21. Rathouska J, Jezkova K, Nemeckova I and Nachtigal P: Soluble endoglin, hypercholesterolemia and endothelial dysfunction. Atherosclerosis 243: 383-388, 2015.

22. van Laake LW, van den Driesche S, Post S, Feijen A, Jansen MA, Driessens MH, Mager JJ, Snijder RJ, Westermann CJ, Doevendans PA, et al: Endoglin has a crucial role in blood cell-mediated vascular repair. Circulation 114: 2288-2297, 2006.

23. Livak KJ and Schmittgen TD: Analysis of relative gene expression data using real-time quantitative PCR and the 2(-Delta Delta C(T)) method. Methods 25: 402-408, 2001.

24. Haseloff RF, Dithmer S, Winkler L, Wolburg H and Blasig IE: Transmembrane proteins of the tight junctions at the blood-brain barrier: Structural and functional aspects. Semin Cell Dev Biol 38: 16-25, 2015.

25. Tornavaca O, Chia M, Dufton N, Almagro LO, Conway DE, Randi AM, Schwartz MA, Matter K and Balda MS: ZO-1 controls endothelial adherens junctions, cell-cell tension, angiogenesis, and barrier formation. J Cell Biol 208: 821-838, 2015.

26. Chadjichristos CE and Kwak BR: Connexins: New genes in atherosclerosis. Ann Med 39: 402-411, 2007.

27. Rennick RE, Connat JL, Burnstock G, Rothery S, Severs NJ and Green CR: Expression of connexin43 gap junctions between cultured vascular smooth muscle cells is dependent upon phenotype. Cell Tissue Res 271: 323-332, 1993.

28. Collins NT, Cummins PM, Colgan OC, Ferguson G, Birney YA, Murphy RP, Meade G and Cahill PA: Cyclic strain-mediated regulation of vascular endothelial occludin and ZO-1: Influence on intercellular tight junction assembly and function. Arterioscler Thromb Vasc Biol 26: 62-68, 2006.

29. Zhu HQ, Wang XB, Han JX, Hu ZP, Wang Y, Zhou Q, Gui SY and Wang Y: Myosin light chain kinase inhibitor attenuates atherosclerosis and permeability via reduced endothelial tight junction in rabbits. Int J Cardiol 168: 5042-5043, 2013. 